\title{
Effect of Vitamin C Supplementation on Lipid Peroxidation and Lactate Levels in Individuals Performing Exhaustion Exercise
}

\author{
${ }^{1}$ Suleyman Patlar ${ }^{*},{ }^{2}$ Abdulkerım Kasım Baltaci, ${ }^{2}$ Rasim Mogulkoc, ${ }^{3}$ Mehmet Gunay \\ ${ }^{1}$ Faculty of Sport Sciences, Selcuk University, Konya,Turkey. ${ }^{2}$ Department of Physiology, Medical School, Selcuk \\ University, Konya, Turkey. ${ }^{3}$ School of Physical Education and Sports, Gazi University, Ankara, Turkey.
}

\begin{abstract}
Background. Exercise has significant affects on free radical production. Objective. The objective of the present study was to explore the effect of vitamin $\mathrm{C}$ supplementation on lipid peroxidation and lactate levels in sedentary individuals who performed exhaustion exercised. Methods. 10 healthy sedentary male athletes participated to study. They were supplemented with $300 \mathrm{mg}$ oral vitamin C (L-ascorbic acid, Ester-C Plus $500 \mathrm{mg}$, Solgar-USA) and subjected to acute exhaustion exercise by fatigue developed one time a week for 4 weeks. Exhaustion exercises were carried out until exhaustion developed according to Bruce protocol once a week for 4 weeks. Blood samples were collected from the subjects four times, at the beginning and at the end of the supplementation and before and after exercise, over the 4-weeks study period. The samples were used to analyze MDA, GSH, GSH-Px, CAT, SOD and NO levels by using ELISA colorimetric method and plasma lactate levels by an autoanalyzer device. Results. Exhaustion MDA, GSH, GSH-Px, CAT, SOD and NO levels measured both before and after supplementation were higher than resting levels $(\mathrm{p}<0.05)$. MDA, NO and lactate levels that were elevated in exhaustion before supplementation decreased significantly after vitamin $\mathrm{C}$ supplementation $(\mathrm{p}<0.05)$. Conclusion. The results of our study indicate that the increase in free radical production and lactate levels due to by 4 -week exhaustion exercise were offset by vitamin $\mathrm{C}$ supplementation. It can be argued in conclusion that supplementation of physiologic doses of vitamin $\mathrm{C}$ may be useful for athlete health and performance.
\end{abstract}

KEY WORDS: Vitamin C, Exercise, Free Radicals, Antioxidant Activity, Lactate.

\section{INTRODUCTION}

Many researchers concentrate on the relation between nutrition on one hand and development and maintaining performance on the other (1-3). Two methods are commonly used to determine the interaction between physical activity and nutrition. The first consists of administering nutrients with various contents to people engaged in physical activity and examining their physiologic and performance responses, while the other involves determining the effects of physical activity on nutrition $(4,5)$. Physical activity and exercise performance may be enhanced by optimal nutrition. Therefore, athlete health and athletic performance require mineral and vitamin support $(2,3)$. Vitamins are primary regulators of metabolic functions which play a critical role in exercise performance (6). Research showed that vitamin deficiency has a

*. Corresponding Author:

Suleyman Patlar

E-mail: s.patlar@yahoo.com.tr 
negative impact on performance, while treatment of the deficiency restores impaired performance $(6,7)$. It was reported that fat-soluble vitamins were crucial to sustain performance and to maintain weight in athletes doing weight-loss exercise $(2,3)$. It was noted that mineral and vitamin support was necessary for a better performance in athletes and also that delaying fatigue and maintaining performance particularly necessitated this kind of supplementation (8). Thus, it can be postulate that there is a growing interest in research into the relationships between exercise, vitamins, minerals and elements.

One of the principal vitamins that need to be supplemented is vitamin C (4). It was argued that because of its role in binding and carrying oxygen and in the absorption of iron by the intestines, vitamin $\mathrm{C}$ administration might be useful in endurance sports in particular $(2,3,5,8,9)$. Vitamin C supplementation was reported to increase systemic and pulmonary antioxidant capacity and prevented oxidative damage and improved pulmonary function during exercise (6). Research into the effects of vitamin $\mathrm{C}$ supplementation to athletes on antioxidant activity and serum iron during the training period and competition season revealed that vitamin $\mathrm{C}$ supplementation prevented the decrease in antioxidant capacity and serum iron during exercise (7). The same study drew attention to a possible relationship between iron metabolism, oxidative stress and vitamin C (7). That vitamin $\mathrm{C}$ supplementation was reported to increase superoxide dismutase (SOD) activity by $20 \%$ also points to the importance of vitamin C in antioxidant activity (10).

Based on an overall evaluation of the current body of knowledge on this topic, it can be argued that there is an inevitable relation between vitamin $\mathrm{C}$ and physical performance and athletes's health. The present study was aimed to explore the effect of vitamin $\mathrm{C}$ supplementation on lipid peroxidation and lactate levels in individuals who were subjected to exhaustion exercise.

\section{MATERIALS AND METHODS}

Informed consent was obtained from each subject included in the study and the study protocol conforms to the ethical guidelines of the
1975 Declaration of Helsinki as reflected in a priori approval by the institution's human research committee the Ethics Board of Selcuk University School of Physical Education and Sports.

Participants. 10 healthy male students of Selcuk University were registered for the study who are not actively engaged in any branch of sports. The mean age of the subjects was found $21.55 \pm 0.22$ years and mean body weight was found $67.89 \pm 2.16 \mathrm{~kg}$. The subjects were supplemented with $300 \mathrm{mg}$ oral vitamin C (Lascorbic acid, Ester-C Plus $500 \mathrm{mg}$, SolgarUSA) and subjected to exhaustion exercise by fatigue set in once-a week for 4 weeks.

In our study, to determine the clear affect of vitamin $C$, we used same subjects to compare before and supplementation parameter, instead of different control group.

Vitamin C Supplementation. Vitamin C (Lascorbic acid, Ester-C Plus $500 \mathrm{mg}$, SolgarUSA)) was administered in tablet form orally at a dose of $300 \mathrm{mg}$ at 10.00 on a full stomach daily for 4 weeks.

Acute Exhaustion Exercice (Bruce Protocole). Exhaustion exercises were performed once a week for 4 weeks in the form of acute exercises until fatigue developed (totally 4 exhaustion were done in which each time for every week). As exhaustion exercise, Bruce protocol (Cosmed T150 treadmill) was applied. In this most commonly used clinical exercise test, slope and speed was increased in 3-minute periods until the subjects grew too tired to continue. Exercise was performed on a treadmill. The treadmill was started at $2.74 \mathrm{~km} / \mathrm{hr}(1.7$ $\mathrm{mph}$ ) and at a gradient (or incline) of $10 \%$. At three minute intervals the incline of the treadmill increases by $2 \%$ and the speed increases. The test was stopped when the subject cannot continue due to fatigue or pain, or due to many other medical indications.

Blood Samples Collection. Blood samples $(5 \mathrm{ml})$ were collected from the forearm vena of the subjects at 09.00 (at fasting period) in the morning four times, during rest and exhaustion before and after the 4-weeks supplementation. The samples were centrifuged at 3000 rotations for 10 minutes to separate the serum which was then stored at $-80^{\circ} \mathrm{C}$ until the analyses. 
Biochemical Analyses. Serum MDA analysis was ELISA colorimetric method conducted by using Cayman commercial kits (catalogue no: 705002). The results were expressed as $\mathrm{nmol} / \mathrm{ml}$. Serum GSH analysis were carried out using Cayman ELISA colorimetric method (catalogue no: 7003002). The results were determined as $\mathrm{mg} / \mathrm{dl}$. Serum Glutathione Peroxidase (GPx) was analyzed using Cayman (catalogue no: 703102) commercial kits by ELISA colorimetric method. The results were presented as $\mathrm{nmol} / \mathrm{ml}$. Serum Superoxide Dismutase (SOD) analyses were performed by using Cayman brand commercial kits and ELISA colorimetric method (catalogue no: 706002). The results were expressed as U/ml. Serum Nitric Oxide (NO) assays were carried out using commercial kits by ELISA colorimetric method (catalogue no: 780001). The results were presented as $\mu \mathrm{M}$. Serum Catalase analyses were conducted using Cayman brand (catalogue no: 707002) commercial kits by ELISA colorimetric method. The results were determined as $\mathrm{nmol} / \mathrm{ml}$. Plasma lactate was analyzed by a lactate analyzer (VARIO Fotometer, Germany) in the blood samples collected into heparinized capillary tubes from the earlobe. The results were expressed as $\mathrm{nmol} / \mathrm{ml}$.

Data Analysis. Statistical evaluation of the findings was carried out by using a computer software package. Arithmetic means and standard deviations of all parameters were calculated. Variance Analysis in Repeated Measurements was employed to detect the differences between measurements performed at different times. $p<0.05$ was considered to significant as statistic.

\section{RESULTS}

The highest and the most important MDA level was obtained before vitamin C supplementation during exhaustion in the present study $(\mathrm{p}<0.05) \quad($ Table 1). There was no significant difference between resting MDA levels before and after vitamin supplementation (p>0.05) (Table 1), but post-supplementation exhaustion value was found significantly lower than the pre-supplementation exhaustion value $(\mathrm{p}<0.05)$ (Table 1).

Exhaustion GSH, GPx, SOD and CAT values before and after vitamin supplementation were significantly higher than resting GSH, GPx, SOD and CAT values $(\mathrm{p}<0.05)$ (Table 1). While resting GSH, GPx, SOD and CAT values did not differ before and after vitamin supplementation ( $>0.05)$ (Table 1), exhaustion values after supplementation were also found similar $(\mathrm{p}<0.05)$ (Table 1).

Exhaustion nitric oxide values of the subjects before and after vitamin supplementation were established to be significantly higher than resting nitric oxide values in our study $(\mathrm{p}<0.05)$ (Table 1). Resting nitric oxide values pre and postsupplementation were similar $(\mathrm{p}>0.05)$ (Table 1). Vitamin $\mathrm{C}$ supplementation significantly reduced exhaustion nitric oxide levels in comparison to pre-supplementation exhaustion levels $(\mathrm{p}<0.05)($ Table 1$)$.

Exhaustion lactate values of the subjects were significantly higher than resting lactate values, as expected as a result of acute exercise $(p<0.05)$ (Table 1). Similarly, 4-week vitamin C supplementation did not change resting lactate values ( $p>0.05)$ (Table 1). However, 4-week vitamin $\mathrm{C}$ supplementation significantly reduced exhaustion lactate values.

\section{DISCUSSION}

Although it is recommended for a healthy lifestyle, moderate exercise is known to lead to oxidative stress, inflammation and muscle injury. Hence, the intense effort to develop dietary strategies to counter the oxidative stress caused by physical activity (11). It has been known that Vitamin $\mathrm{C}$ has preventive role in tissue damage which is occurred by free radicals, thereafter it has been postulated that may prevents oxidative damage in sportsmen (12). Nakhostin-Roohi et al. (13) have reported that single dose $1000 \mathrm{mg}$ vitamin C prevented lipid peroxidation in sportsmen. Similarly, $1000 \mathrm{mg}$ vitamin $\mathrm{C}$ supplementation has been useful affects in sportsmen (14). In clinical trials, it has been shown that vitamin $C$ supplementation may be done by $1000 \mathrm{mg}$ for 60 days at humans (15). Thereafter, we have supplemented vitamin $\mathrm{C}$ as $300 \mathrm{mg}$ /day for 1 month (16).

Pre- and post-vitamin supplementation exhaustion MDA values were found significantly higher than resting MDA levels in the present study. Physical activity brings about an increase in oxidative stress, muscle tissue 
damage, lipid peroxidation in the membranes and formation of free radical products. Oxidative stress that develops parallel to increased oxygen consumption during exercise is considered the major cause of elevated free radical production (17). Increased MDA values we obtained in exhaustion result from acute exercise are parallel to the reports on this topic (17). However, 4weeks vitamin $C$ supplementation significantly reduced exhaustion MDA values relative to the pre-supplementation exhaustion values. This result indicates that vitamin $\mathrm{C}$ supplementation inhibits free radical production in individuals engaged in physical activity. Optimal nutrition is essential for recuperation following physical activity and exercise. Nutrient supplementation is recommended for athlete health and performance in high-intensity sports branches. At athletes, exercises that lead to a decrease in body weight in particular need supplementation of water- and fat-soluble vitamins $(2,3)$. One of the key vitamins that should be supplemented is vitamin C (18). It is reported that vitamin C which is of utmost importance in the absorption of iron from the digestive system can boost performance especially in individuals who done endurance sports (19-22). The results of our study concerning the MDA parameter indicate that physical activity brought about an increase in free radical production, whereas vitamin $\mathrm{C}$ supplementation reduced the production of free radicals. These results we obtained with vitamin C supplementation are consistent with the results of the researchers cited above.

\begin{tabular}{|c|c|c|c|c|}
\hline \multicolumn{5}{|c|}{ Table 1. Biochemical Parameters in the Study Subjects (mean $\pm s d ; n=10)$} \\
\hline \multirow{2}{*}{$\begin{array}{l}\text { Antioxidant } \\
\text { Parameters }\end{array}$} & \multicolumn{2}{|c|}{ before vitamin $\mathrm{C}$ supplementation } & \multicolumn{2}{|c|}{ after vitamin $\mathrm{C}$ supplementation } \\
\hline & Resting (1) & Exhaustion (2) & Resting (3) & Exhaustion (4) \\
\hline MDA (nmol/ml) & $9.48 \pm 0.86^{\mathrm{C}}$ & $15.16 \pm 0.64^{\mathrm{A}}$ & $8.62 \pm 1.27^{\mathrm{C}}$ & $11.68 \pm 1.17^{\mathrm{B}}$ \\
\hline GSH (mg/dl) & $20.90 \pm 2.74^{\mathbf{B}}$ & $30.04 \pm 4.71^{\mathrm{A}}$ & $22.64 \pm 4.02^{\mathrm{B}}$ & $32.08 \pm 4.34^{\mathrm{A}}$ \\
\hline GSH-Px(nmol/ml) & $50.30 \pm 1.87^{\mathrm{B}}$ & $60.65 \pm 2.65^{\mathrm{A}}$ & $49.08 \pm 1.31^{\mathrm{B}}$ & $63.54 \pm 8.43^{\mathrm{A}}$ \\
\hline SOD $(\mathbf{U} / \mathbf{m l})$ & $0.03 \pm 0.006^{\mathbf{B}}$ & $0.07 \pm 0.009^{\mathbf{A}}$ & $0.04 \pm 0.006^{\mathbf{B}}$ & $0.08 \pm 0.006^{\mathbf{A}}$ \\
\hline NO $(\mu \mathrm{M})$ & $5.18 \pm 0.73^{\mathrm{C}}$ & $12.28 \pm 2.56^{\mathrm{A}}$ & $4.92 \pm 0.55^{\mathrm{C}}$ & $9.22 \pm 1.08^{\mathbf{B}}$ \\
\hline CAT(nmol/ml) & $10.04 \pm 2.04^{\mathbf{B}}$ & $14.90 \pm 1.68^{\mathrm{A}}$ & $9.28 \pm 1.37^{\mathbf{B}}$ & $15.88 \pm 2.54^{\mathrm{A}}$ \\
\hline Lactate $(\mathrm{mmol} / \mathrm{l})$ & $1.41 \pm 0.20^{\mathrm{C}}$ & $10.33 \pm 1.42^{\mathrm{A}}$ & $1.38 \pm 0.21^{\mathrm{C}}$ & $7.71 \pm 1.56^{\mathbf{B}}$ \\
\hline
\end{tabular}

A,B,C: Differences between means with different superscripted letters in the same line are statistically significant $(\mathrm{p}<0.05)$.

P values;

MDA: $1-2=0,000 ; 1-3=0,735 ; 1-4=0,000 ; 2-3=0,000 ; 2-4=0,002 ; 3-4=0,000$

GSH: $1-2=0,000 ; 1-3=0,669 ; 1-4=0,000 ; 2-3=0,001 ; 2-4=0,805 ; 3-4=0,000$

GSH-Px: $1-2=0,000 ; 1-3=0,153 ; 1-4=0,000 ; 2-3=0,000 ; 2-4=0,200 ; 3-4=0,000$

SOD: $1-2=0,000 ; 1-3=0,624 ; 1-4=0,000 ; 2-3=0,002 ; 2-4=0,605 ; 3-4=0,000$

NO: $1-2=0,000 ; 1-3=1,000 ; 1-4=0,000 ; 2-3=0,000 ; 2-4=0,001 ; 3-4=0,000$

CAT: $1-2=0,000 ; 1-3=1,000 ; 1-4=0,000 ; 2-3=0,000 ; 2-4=0,824 ; 3-4=0,000$

Lactate: $1-2=0,000 ; 1-3=0,155 ; 1-4=0,000 ; 2-3=0,000 ; 2-4=0,021 ; 3-4=0,000$

In the present study, exhaustion GSH, GPx, SOD and CAT values of the subjects were found significantly higher than resting GSH, GPx, SOD and CAT values. Levels of antioxidants are known to vary depending on the intensity, type and length of exercise (23). However, it was reported that the activity of antioxidant enzymes like SOD and catalase in the blood and tissues increased after acute exercise (24). Exercise may be increase the antioxidant defense in general. Previous studies established post-exercise increases in the erythrocyte GSH, catalase and glutathione reductase (25). There are also reports of regular exercise and training stimulating the biosynthesis of antioxidant enzymes (26). Therefore, regular exercise is recognized to arise as a critical adaptation mechanism in reducing the oxidative stress following acute exercise (27). This condition may be explained by the good adaptability habits of the antioxidant defense systems of athletes. Plasma ascorbic acid, alpha-tocopherol, uric acid and total GSH levels in regularly training elite 
female football players who played a 40-minute football game were found to increase immediately after the game. This increase in the antioxidant defense may be attributed to the transfer of endogenous antioxidants and/or antioxidants accumulated in the tissues to circulation with exercise (28). It was reported in an experimental animal study that the increase in free radical formation by exercise was in much more and that free radical formation could not be prevented despite the increase in the antioxidant system (23). Elevated exhaustion antioxidant parameters we obtained before supplementation in our study are consistent with the reports of the above-cited researchers. The increase in the exhaustion antioxidant parameters occurs possibly as an adaptation mechanism designed to prevent the increase in free radical production. All the reports cited above support to the elevated antioxidant parameters we found after exercise.

Pre-and post-supplementation exhaustion nitric oxide values of the subjects were significantly higher than the resting nitric oxide values in the present study. NO is a lowmolecular-weight, lipophilic free radical gas. It reacts rapidly with the oxygen in the medium and is converted to toxic nitrogen dioxide $\left(\mathrm{NO}_{2}\right)$ (29). The reaction between NO and superoxide results in the generation of peroxynitrite $\left(\mathrm{ONOO}^{-}\right)$, a reactive oxidant, which in turn reacts with tyrosine to generate 3-nitrotyrosine (30). We could not find a study with which to compare ours directly in terms of the relation between nitric oxide and exercise. However, the fact that nitric oxide (NO) was shown to regulate the vessel tonus and to cause vasodilation after exercise (31). At least may be an explain for the elevated nitric oxide levels we obtained after exercise in our study. That exercise was reported to play a key stimulating effect on the production of nitric oxide by causing an increase in blood flow (32). Noteworthy piece of information supporting the elevated NO finding in our study.

Four-week vitamin $\mathrm{C}$ supplementation in our study significantly reduced exhaustion NO values when compared to the presupplementation exhaustion values. This result is important, because low concentrations of nitric oxide are involved in physiological processes like the control of vascular tonus, neurotransmission, learning and memory (33), while high concentrations rapidly react with the oxygen in the medium to transform into toxic nitrogen dioxide $\left(\mathrm{NO}_{2}\right)$ (29). That vitamin $\mathrm{C}$ supplementation significantly reduced exhaustion NO levels relative to presupplementation exhaustion levels may add substance to the supplementation of vitamins in athlete nutrition and performance.

As expected, exhaustion lactate values of the subjects were significantly higher than resting values in this study. Similarly, 4-weeks vitamin $\mathrm{C}$ supplementation did not change resting lactate values. However, a significant decrease was found in the exhaustion lactate values following vitamin $\mathrm{C}$ supplementation. Vitamin $\mathrm{C}$ is one of the leading vitamins that should be supplemented for athlete performance and health (4). Given its indirect role in meeting the tissues' oxygen needs, it is no surprise that vitamin $\mathrm{C}$ is associated with fatigue. Decreased lactate values we found in the exhaustion period after vitamin $\mathrm{C}$ supplementation may be quite significant in the relation between vitamin $\mathrm{C}$ and exhaustion and may stimulate research into various aspects of this relation.

\section{CONCLUSION}

The results of our study indicated that vitamin C supplementation prevented the increase in lactate levels and free radical production caused by 4-weeks exhaustion exercise. Vitamin C supplementation in physiological doses may be useful in prevents of tissue damage due to exercise.

\section{APPLICABLE REMARKS}

- Vitamin C supplementation can be prevented the increase in lactate levels and free radical production.

\section{ACKNOWLEDGMENTS}

All authors wish to thank the subjects for their efforts in this study. The authors stated that there are no conflicts of interest regarding the publication of this article. 


\section{REFERENCES}

1. Kilic M. Effect of fatiguing bicycle exercise on thyroid hormone and testosterone levels in sedentary males supplemented with oral zinc. Neuro Endocrinology Letters. 2007;28:681-685.

2. Rodriguez NR, Di Marco NM, Langley S. American College of Sports Medicine position stand. Nutrition and athletic performance. Medicine and Science in Sports and Exercise. 2009a;41:709-231.

3. Rodriguez NR, DiMarco NM, Langley S. Position of the American Dietetic Association, Dietitians of Canada, and the American College of Sports Medicine: Nutrition and athletic performance. Journal of the American Dietetic Association. 2009b;109:509-527.

4. Brotherhood JR. Nutrition and sports. Sports Medicine. 1984;1:350-389.

5. Finstad EW, Newhouse IJ, Lukaski HC, McAuliffe JE, Stewart CR. The effects of magnesium supplementation on exercise performance. Medicine and Science in Sports and Exercise. 2001;33:493-498.

6. Williams MH. Vitamin supplementation and athletic performance. International Journal for Vitamin and Nutrition Research - Supplement. 1989;30:163-191.

7. Kujala UM, Sarna S, Kaprio J. Use of medications and dietary supplements in later years among male former toplevel athletes. Archives of Internal Medicine. 2003;163:1064-1068.

8. Sobal J, Marquart LF. Vitamin/mineral supplement use among high school athletes. Adolescence. 1994;29: 835843.

9. Briefel R, Hanson C, Fox MK, Novak T, Ziegler P. Feeding Infants and Toddlers Study: do vitamin and mineral supplements contribute to nutrient adequacy or excess among US infants and toddlers? The Journal of the American Dental Association. 2006;106:52-65.

10. Aydemir T, Oztürk R, Bozkaya LA, Tarhan L. Effects of antioxidant vitamins A, C, E and trace elements $\mathrm{Cu}, \mathrm{Se}$ on CuZn SOD, GSH-Px, CAT and LPO levels in chicken erythrocytes. Cell Biochemistry \& Function. 2000;18:109-115.

11. Baltaci SB, Mogulkoc R, Baltaci AK. Resveratrol and exercise. Biomed Rep. 2016;5(5):525-530.

12.Popovic LM, Mitic NR, Miric D, Bisevac B, Miric M, Popovic B. Influence of vitamin C supplementation on oxidative stress and neutrophil inflammatory response in acute and regular exercise. Oxid Med Cell Longev. 2015;2015:295497.

13. Nakhostin-Roohi B, Babaei P, Rahmani-Nia F, Bohlooli S. Effect of vitamin C supplementation on lipid peroxidation, muscle damage and inflammation after 30-minexercise at 75\% VO2max. J Sports Med Phys Fitness. 2008;48(2):217-24.

14. Paulsen G, Cumming KT, Hamarsland H, Børsheim E, Berntsen S, Raastad T. Can supplementation with vitamin C and E alter physiological adaptations to strength training? BMC Sports Sci Med Rehabil. 2014;5:6:28.

15. Cengiz M, Cengiz S. The Effects of vitamine $\mathrm{C}$ administration on erythrocyte glutathione and HbAlc levels of type 2 diabetic patients. Cerrahpaşa J Med 2000; 31(4): 211-215.

16. Patlar S. Boyalı E, Baltaci AK, Mogulkoc R, Gunay M. Effect of Vitamin C supplementation on various elements in elite taekwondo players. Medicina dello Sport 2012; 65(1):49-56.

17. Aslan R, Şekeroğlu MR, Tarakçığlu M, Bayıroğlu F. Investigation of Malondialdehyde Formation and Antioxidant Enzyme Activity in Stored Blood. Haematologia.1997;28:18-21.

18. Goldfarb AH, Bloomer RJ, McKenzie MJ. Combined antioxidant treatment effects on blood oxidative stress after eccentric exercise. Medicine and Science in Sports and Exercise. 2005;37:234-239.

19. Shephard RJ. The athlete at high altitude. Canadian Medical Association Journal. 1973;109: 207-209.

20. Masek J, Hruba F. On the relation between the saturation of the serum and the leukocytes with vitamin C. International Journal of Vitamin Research. 1964;34:39-44.

21. Schrauzer GN, Rhead WJ. Ascorbic acid abuse: effects on long term ingestion of excessive amounts on blood levels and urinary excretion. Annals of The New York Academy of Sciences. 1973;43:201-211.

22. Schrauzer GN, Ishmael D, Kiefer GW. Some aspects of current vitamin C usage: diminished high-altitude resistance following overdosage. Annals of The New York Academy of Sciences. 1975;258:377-381.

23. Fiçıcılar H, Zergeroglu AM, Tekin D, Ersoz G. The effects of acute exercise on plasma antioxidant status and platelet response. Thrombosis Research. 2003;111:267-71.

24. Banerjee AK, Mandal A, Chanda D, Chakraborti S. Oxidant, antioxidant and physical exercise. Molecular and Cellular Biochemistry. 2003;253:307-12.

25. Clarkson PM, Thompson HS. Antioxidants: what role do they play in physical activity and health? The American Journal of Clinical Nutrition. 2000;72:637-646.

26. Viña J, Gomez-Cabrera MC, Lloret, Marquez R, Miñana JB, Pallardó FV, Sastre JA. Free radicals in exhaustive physical exercise: mechanism of production, and protection by antioxidants. IUBMB Life. 2000; 50:271-277. 
27. Brites FD, Evelson PA, Christiansen MG Nicol MF, Basilico MJ, Wikinski RW, Llesuy SF. Soccer players under regular training show oxidative stress but an improve plasma antioxidant status. Clinical Science. 1999;96:381385.

28. Andersson H, Karlsen A, Blomhoff R, Raastad T, Kadi F. Plasma antioxidant responses andoxidative stress following a soccer game in elite female players. Scandinavian Journal of Medicine \& Science in Sports. 2010;20:600-608.

29. Tschakovsky ME, Joyner MJ. Nitric oxide and muscle blood flow in exercise. Applied Physiology, Nutrition, and Metabolism. 2008;33:151-61.

30. Drew B, Leeuwenburgh C. Aging and the role of reactive nitrogen species. Annals of The New York Academy of Sciences. 2002;959:66-81.

31. Gilligan DM, Panza JA, Kilcoyne CM Waclawiw MA, Casino PR, Quyyumi AA. Contribution of endotheliumderived nitric oxide to exercise induced vasodilation. Circulation. 1994;90:2853-2858.

32. Shen W, Zhang X, Zhao G, Wolin MS, Sessa W, Hintze TH. Nitric Oxide production and NO Synthase gene expression contribute to vascular regulation during exercise. Medicine and Science in Sports and Exercise. 1995;27:1125-1134.

33. Sezer Ö, Karlıdağ R, Karabulut AB, Özcan C, Nisaoğlu V, Türköz Y, Bulut A, Ünal S. Relationship between nitric oxide levels and delirium in patients with coronary bypass operation. Bulletin of Clinical Psychopharmacology. 2004;14:185-190. 